\title{
Efficient Skeleton Editing in a VR Environment Facilitates Accurate Modeling of Highly Branched Mitochondria.
}

Ryan Conrad ${ }^{1}$, Thomas Ruth ${ }^{2}$, Falko Löffler ${ }^{2}$, Steffen Hadlak ${ }^{2}$, Sebastian Konrad ${ }^{2}$, Christian Götze ${ }^{2}$, Christopher Zugates ${ }^{3}$, Molly McQuilken ${ }^{3}$ and Kedar Narayan ${ }^{1}$

${ }^{1}$ National Cancer Institute, NIH \& Frederick National Laboratory for Cancer Research, Frederick, Maryland, United States, ${ }^{2}$ arivis AG, Rostock, Mecklenburg-Vorpommern, Germany, ${ }^{3}$ arivis inc., Washington, District of Columbia, United States

Mitochondria are ubiquitous organelles involved in a variety of essential processes in eukaryotic cells, readily recognized by electron microscopy (EM) on account of their double-membrane-bound structure and internal membrane folds called cristae. Contrary to textbook cartoons, mitochondria display widely different morphologies depending on many variables such as cell type and disease status [1]. These variations are not limited to any 2D plane and can be interrogated with high-resolution 3D fluorescence microscopy and EM techniques. Volume EM approaches such as focused ion beam scanning electron microscopy (FIB-SEM) have the resolution in all three axes to visualize fine ultrastructural changes in mitochondria. However, creating accurate representations for visual and quantitative analysis continues to be a challenge. Automated or semi-automated approaches to delineate these complex morphologies in a crowded intracellular context still require manual intervention to ensure model fidelity [2]. For example, "skeletonization" algorithms mathematically reduce 3D shapes into a series of linked points in space, called a "curve skeleton", that roughly adhere to the centerlines of an object's branches. Close inspection of skeletons often reveals errors such as poor centering, spurious branches, or broken topologies - these errors profoundly alter downstream analyses and hobble efforts at automated quantification [3]. Yet manual clean-up of skeletons is slow, non-intuitive, and prone to subjective errors because of a central limitation - the interaction with 3D data on a 2D plane (computer monitor or drawing tablet), a constraint inextricably associated with information loss and a reduced awareness of spatial relationships.

The application of virtual reality (VR) to visualize volumetric image data has a long history, beginning with displays of molecular models and astrophysical data [4]. Dramatic improvements in hardware and software solutions have opened avenues for VR applications in other scientific disciplines such as 3D microscopy, which often generates massive image datasets [5]. Volume rendering itself is a computationally demanding task and VR rendering requires double the number of calculations for every corresponding stereo-image. Moreover, these calculations must be done in real-time to provide smooth viewing and interaction with the data. To overcome these challenges, iso-surface rendering or projection rendering techniques have been used to achieve acceptably fast generation of stereo-images [6]. In our approach, we introduced an agile ray-tracing technique in conjunction with custom smoothing operations and a quick-access data format to achieve fast, high quality VR rendering of 3D microscopic image data on commercially available Oculus Rift headsets [7].

In addition to visualization, VR rendering of volumetric data can help to validate and improve the accuracy and efficiency of quantifications [8]. Here, we developed tracing functionalities to analyze and correct the curve skeletons of complex objects and their extensions, specifically, mitochondrial networks in FIB-SEM volume reconstructions of mammalian cells. Previously, we generated FIB-SEM image volume reconstructions of fixed, stained, and resin-embedded mammalian cells according to established protocols [9]. These image volumes contained a number of convoluted and branched mitochondria that we wished to characterize. We utilized the skeletonize_3d function provided in scikit-image to create curve skeletons 
from the label maps (although other skeletonization algorithms may also be used) [10]. The voxel skeletons were separated into branches at the node points where they were connected. Each branch was then converted into a set of ordered 3D coordinates and stored in a .csv file for import into the VR environment, along with the label maps corresponding to the mitochondria themselves. This allowed us to interact with mitochondrial reconstructions and associated skeletons at the same time and in context (Figure 1). For clarity, we display the results from a sub-volume containing five complete mitochondria of different sizes and shapes. There were a variety of errors generated by the skeletonization module, including spurious branch connections and extraneous bumps or "jitters" arising from underlying mathematical boundary conditions - these were easily and intuitively visualized. In addition to skeleton visualization in VR, we developed modules that then allow for easy addition, deletion and editing of points to alter the skeleton appropriately within the environment; these corrected skeleton coordinates can be exported again as a .csv file for downstream analysis. The connectivity map generated with this tool can thus be used to quantify various aspects of other complex cellular networks, including datasets of neuronal processes acquired by LM or EM approaches.

The process of manually editing $3 \mathrm{D}$ data on a computer monitor or tablet is hindered by the inherent shortcomings of having to project these data on a 2D plane. With VR, users now have the unique potential to use their hands to navigate through a 3D image and intuitively edit data within 3D space; this simplifies and speeds up manual proofreading of automated approaches to object modeling and creates opportunities for novel applications. 

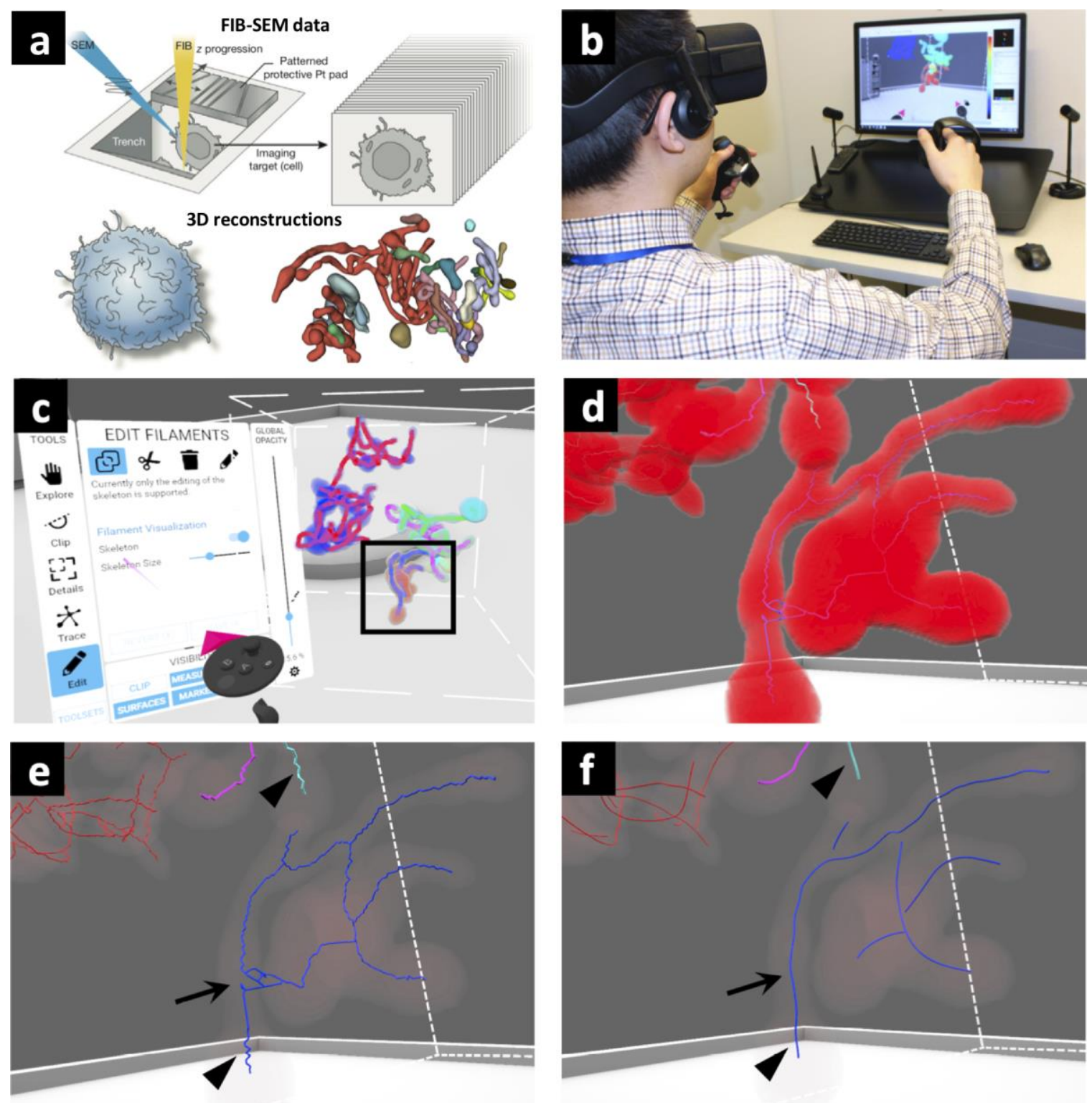

Figure 1. (a) FIB-SEM imaging of mammalian cells resulting in nanoscale resolution volume reconstructions of mitochondrial networks. (b) Mitochondrial volume renderings as imported into a VR environment for intuitive 3D interaction. (c) Algorithm-derived curve skeletons of mitochondria for downstream characterization as visualized in VR. A subset of mitochondria skeletons (thick lines) are shown for clarity. (d) An individual volume rendered mitochondria, boxed in (c), as displayed in VR; closer inspection of the skeleton reveal errors that are challenging to correct with $2 \mathrm{D}$ visualization. (e) Two common sets of errors that occur from skeletonization (curve skeletons, blue) before VR based correction: spurious branches (arrow) and jitters (arrowheads) in the tracings. The mitochondrion is rendered at high transparency to guide the eye. (f) Same skeleton after manual fixing of errors in the VR environment. Errors are typically fixed within minutes, depending on complexity of the skeleton.

\section{References}

[1] Vincent, A. E., White, K., Davey, T., Taylor, R. W., Turnbull, D. M., \& Picard, M. (2019). Quantitative 3D Mapping of the Human Skeletal Muscle Mitochondrial Network. Cell Reports 26(4), 996-1009. 
[2] Kasthuri, N., Hayworth, K. J., Berger, D. R., Schalek, R. L., Conchello, J. A., Knowles-Barley, S., ... Lichtman, J. W. (2015). Saturated Reconstruction of a Volume of Neocortex. Cell 162(3), 648-66.

[3] Tagliasacchi, A., Delame, T., Spagnuolo, M., Amenta, N., Alexandru, T. (2016) 3D Skeletons: A State-of-the-Art Report. Computer Graphics Forum 35 (2), 573-597.

[4] Göbel M., Müller H., Urban B. (1995): Visualization in scientific computing. Springer-Verlag.

[5] Huisken, J., Swoger, F., Del Bene, J., Wittbrodt, E., Stelzer, H. (2004). Optical sectioning deep inside live embryos by selective plane illumination microscopy. Science 305(5686), 1007-1009.

[6] Costantini, M. , Ambrosini, E. Scorolli, C. et al. (2011). When objects are to close to me: Affordances in the peripersonal space. Psychon Bull Rev 18, 302.

[7] Löffler, F., Bönisch, B., Götze, C., Suchanek, A.: Direct Volume Rendering in Virtual and/or Augmented Reality, 20170109935. Filed: October 17, 2016 \& issued April 20, 2017; 10319147. Filed October 17, 2016 \& issued June 11, 2019; 20190272027. Filed May 21, 2019 \& issued Spetember 5, 2019.

[8] Wang, Y., et al. (2019) TeraVR empowers precise reconstruction of complete 3-D neuronal morphology in the whole brain. Nature communications 10, 3474.

[9] Rahman, M., Chang, I. Y., Harned, A., Maheshwari, R., Amoateng, K., Narayan, K., \& Cohen-Fix, O. (2020). C. elegans pronuclei fuse after fertilization through a novel membrane structure. The Journal of Cell Biology. 219(2), e201909137.

[10] Stéfan van der Walt, Johannes L. Schönberger, Juan Nunez-Iglesias, François Boulogne, Joshua D. Warner, Neil Yager, Emmanuelle Gouillart, Tony Yu and the scikit-image contributors. (2014). scikitimage: Image processing in Python. PeerJ 2, e453. 